\title{
Estimating the chromospheric magnetic field from a revised NLTE modeling: the case of HR 7428
}

\author{
Innocenza Busá ${ }^{1}$ \\ ${ }^{1}$ INAF - Catania Astrophysical Observatory, \\ Via S. Sofia, 78, 95123 \\ - Catania - Italy \\ email: innocenza.busa@oact.inaf.it
}

\begin{abstract}
Semi-empirical atmospheric modeling is here used to obtain the chromospheric magnetic field distribution versus height in the K2 primary component of the RS CVn binary system HR 7428. The chromospheric magnetic field estimation versus height comes from considering the possibility of not imposing hydrostatic equilibrium in the atmospheric modeling. The stability of the best Non-hydrostatic equilibrium model, implies the presence of and additive (toward the center of the star) pressure, that decrease in strength from the base of the chromosphere toward the outer layers. Interpreting the additive pressure as magnetic pressure and I derive a magnetic field intensity of about 500 Gauss at the base of the chromosphere.
\end{abstract}

Keywords. magnetic field, stars: chromospheres, stars: modeling atmosphere, stars: activity

\section{Introduction}

HR $7428(=\mathrm{V} 1817 \mathrm{CYgni})$ is a bright $(\mathrm{V}=6.3)$ long-period (108.578d) spectroscopic RS CVn binary composed by a K2 II-III star and a main sequence A2 star (Parsons \& Ake 1987). The magnetic activity of the system is well known: Ca II H \& K emission was firstly reported by Gratton (1950), by a detailed analysis of photometric observations Hall et al. (1990) were able to detect starspot signatures on the K2 primary star. Is now well established that stellar atmospheres of cool stars are characterized by a temperature gradient inversion. That is explained inside a magnetic activity theory, but not yet definitively understood. Late-type stars with $\mathrm{H}-\alpha$ in emission show a fairly stable chromospheric emission outside flares (see. e.g., Byrne et al. 1998). This reinforces the hypothesis that chromospheres are globally in a quasi-stationary state, modulated mainly by the stellar activity cycle, whose temperature-density structure results from the balance between global dissipation of non-radiative energy and radiative cooling (see Kalkofen et al. 1999).

Most of what we know about stars and systems of stars is derived from an analysis of their radiation and this knowledge will be secure only as long as the analytical technique is physically reliable. A well tested technique to get information on physical properties of chromospheric layers of active stars is the NLTE radiative transfer semi-empirical modeling: for different temperature vs.height distributions, the NLTE populations for hydrogen are computed, solving simultaneously the equations of hydrostatic equilibrium, radiative transfer and statistical equilibrium. The emerging profiles for some chromospheric lines and continua are computed and compared to the observations. Then, the modeling is iterated until a satisfactory match is found. (see, e.g., Vernazza et al. 1981; Fontenla et al. 1993). These models are built to match the observations in different spectral features, and make no assumption about the physical processes responsible for the heating of the 
chromosphere, but can be used as constraints for these processes. The obtained models describe the variations of the essential physical parameters, in particular the temperature, pressure and electron density across the outer atmosphere, and give information on its "mean" state, both temporally and spatially.

The most important problem of this approach lies in the uniqueness of the solution. In fact, knowing that a particular atmosphere would emit a line profile like the one we observe for a given star does not imply that the star has indeed this atmospheric structure, since we do not know whether some other atmospheres would produce the same line profiles. To solve, or at least to reduce, this problem, the modeling has to be based on several spectral features, with different regions of formation. The amount and the kind of diagnostics used to build an atmospheric model is in fact, very important, by combining several spectral lines that are formed at different but overlapping depths in the atmosphere, we can obtain a more reliable model (Mauas et al. 2006).

Certainly the best known semiempirical model is the one for the average Quiet-Sun, Model C by Vernazza et al. (1981). Semiempirical modeling was successfully applied also to the atmospheres of cool stars. An extensive modeling of dM stars, has been done, starting with the work by Cram \& Mullan (1979), Short \& Doyle (1998), and by Mauas \& Falchi (1994), and Mauas et al. (1997). In cool stars the application of NLTE semi-empirical chromospheric modelling can be based on optical and ultraviolet (UV) observations. This is because lines such as the $\mathrm{H}-\alpha$ Na I D Ca II IRT become dominated by electron-collision excitation processes, which make them effective chromospheric diagnostics (Houdebine 1996). The possibility of using H- $\alpha$ profile as a diagnostic of stellar chromospheres was discussed in detail by Cram \& Mullan (1979) and Mullan \& Cram (1982) in terms of control of the source function by collisional processes. H- $\alpha$ is observed in active stars, in a wide variety of shapes and sizes; when the effective temperature is low enough, the collisional control of the $\mathrm{H}-\alpha$ source function become possible over a wide range of chromospheric pressures. Under conditions of collisional control, H- $\alpha$ can be a good chromospheric pressure diagnostic. MgIIh\&k UV lines, due to their large opacity, provide excellent diagnostic over a wide range of heights of the upper chromospheric layers (Uitenbroek 1992), and Ca II IRT triplet is a constraint for the shape of the middle chromosphere from the temperature minimum up to the plateau (Andretta et al. 2005). Here I applied the NLTE semi-empirical chromospheric modelling to the K2 star of HR 7428 binary system basing the analysis on the H- $\alpha$ H- $\beta$ Na I D Ca II IRT triplet and $\mathrm{Mg}$ II h\&k lines and UV continuum diagnostics.

\section{Data acquisition and reduction}

H- $\alpha$ Na I D H- $\beta$ Ca II IRT spectroscopic observations of HR 7428 were carried out at the 91-cm telescope of Catania Astrophysical Observatory, "M. G. Fracastoro" station (Serra La Nave, Mt. Etna, Italy), using the new Catania Astrophysical Observatory Spectropolarimeter (CAOS) which is a fiber fed, high-resolution, cross-dispersed echelle spectrograph (Leone et al. 2016, Spanó et al. 2004, Spanó et al. 2006.)

The spectra were obtained in July 2015. Exposure times have been tuned in order to obtain a signal-to-noise ratio of at least 200 in the continuum in the 390-900 nm, with a resolution of $R=\frac{\Delta(\lambda)}{\lambda}=45,000$, as measured from ThAr and telluric lines.

Echelle IRAF packages have been used for data reduction, following the standard steps: bias subtraction, background subtraction, trimming, flat-fielding, scattered light subtraction and order extraction. Several ThAr lamp exposures were obtained during each night and then used to provide a wavelength calibration of the observations. 
Table 1. HR 7428 K2II-III and A2 components as determined by Marino et al. (2001)

\begin{tabular}{ccc}
\hline Element & Primary (cooler K2II-III) & Secondary (hotter A2) \\
\hline $\mathrm{R}$ & $40.0 \pm 6.5 R_{\odot}$ & $2.25 \pm 0.5 R_{\odot}$ \\
$T_{\text {eff }}$ & $4400 K \pm 150 K$ & $9000 K \pm 200 K$ \\
$\log g$ & $2.0 \pm 0.5$ & $4.0 \pm 0.5$ \\
\hline
\end{tabular}

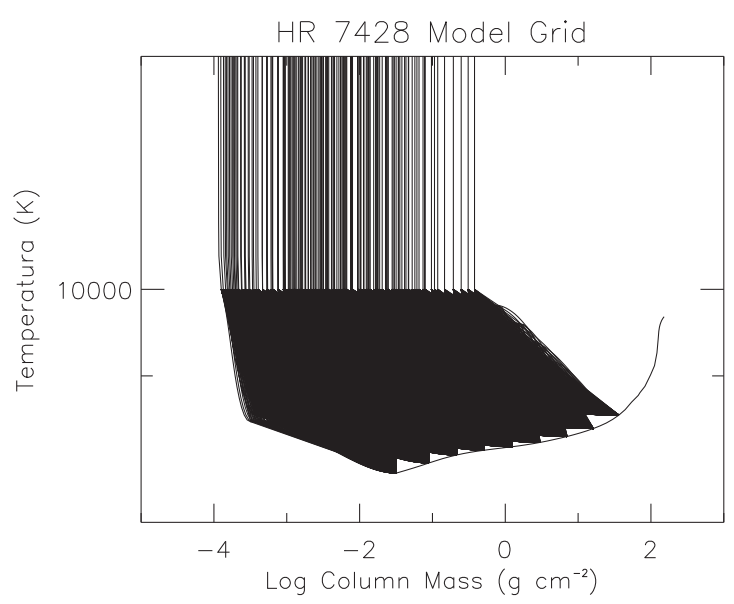

Figure 1. Grid of 15691 models used for the study of HR7428 atmosphere. The grid has been built $\mathrm{s}$ described in the text.

Each spectral order was normalized by a polynomial fit to the local continuum. Mg II h\&k spectroscopic observations have been obtained, in 1997, by the IUE satellite. IUE spectra have been corrected for interstellar extinction. The typical value of 1 magnitude per kilo-parsec for the interstellar extinction and the Hipparcos distance $(\mathrm{d}=323 \mathrm{pc})$, adopted for the computations, lead to an extinction magnitude $\mathrm{A}(\mathrm{V})=$ 0.32 . Assuming the standard reddening law $A(V)=3.1 \times \mathrm{E}(\mathrm{B}-\mathrm{V})$, a color excess $\mathrm{E}(\mathrm{B}-\mathrm{V})=$ 0.10 has been derived. IUE spectra have been de-reddened according to the selective extinction function of Cardelli et al. (1989). The spectral resolution is about $0.2 \AA$ for the $\mathrm{Mg}$ II region.

\section{The model grid}

The atmospheric model of the K2 primary magnetic active component has been built computing a photospheric model, a chromospheric model and a transition region model and joining the three together.

The Photospheric Model

Taking into account the Marino et al. (2001) HR 7428 physical parameters (Table 1) we computed the A2 secondary component atmospheric model, by the ATLAS9 code (Kurucz 1993) using the parameters $\log g=4.0, T_{\text {eff }}=9000 \mathrm{~K}$ and solar metalicity and the K2 primary component photospheric model using the parameters $\log g=2.0, T_{\text {eff }}=$ $4400 \mathrm{~K}$ and solar metalicity. The A star dominate the continuum emission for $\lambda \leqslant 3200$ $\AA$, while for wavelength longer than $\lambda=3200 \AA$, the continuum is dominated by the $\mathrm{K}$ star, therefore I neglected the contribution of the A2 when computing optical line profiles while I took it into account in the computation of the $\mathrm{Mg}$ II h\&k lines. 
A first estimation of a plane-parallel model for the lower transition region of the HR 7428 K2 primary component, has been built using the method of the Volumetric Emission Measure (Jordan \& Brown 1981, Harper 1992). The flux at the star, for lines forming at temperature $T_{e} \approx 10^{5}$ are, dominated by collisions and this results in emission lines that are optically thin and with a contribution function sharply picked in temperature, that is, typically formed over a temperature range of $\Delta \log \left(T_{e}\right)=0.30$. The above conditions allow to derive the temperature gradient as a function of the averaged Emission Measure over $\Delta \log \left(T_{e}\right)=0.30$ that we indicate as $E M_{0.3}$.

By imposing hydrostatic equilibrium, including turbulent pressure, the transition region model can be obtained by combining the temperature gradient as a function of $E M_{0.3}$ and the pressure variation from the equation of hydrostatic equilibrium (see, e.g., Harper 1992). The expression for the temperature gradient combined with the equation of hydrostatic equilibrium gives the relationship:

$$
P_{T}^{2}\left(T_{2}\right)-P_{T}^{2}\left(T_{1}\right)=2 *(1.4)^{2} m_{p} g k \int_{T_{1}}^{T_{2}}\left[E M_{0.3}(1+1.1 x)+\frac{1.4 x m_{p} \eta^{2} E M_{0.3}}{2 k T}\right] d T
$$

where $m_{p}$ is the $H^{+}$mass, $k$ is the Boltzmann constant and $\mathrm{x}=N_{H} / N_{e}$.

The Eq. 3.1 together with an estimate of electron density and turbulent velocity in a layer, allows to find the pressure as a function of temperature for the TR model. The total particle density $\left(N_{t o t}\right)$, gas pressure $\left(P_{G}\right)$, turbulence pressure $\left(P_{\text {turb }}\right)$ and electron density $\left(N_{e}\right)$ are then obtained according to the following relations

$$
\begin{aligned}
& N_{t o t}=\sqrt{P_{T}}\left(k T+0.5 m_{p} \eta^{2}\right) \\
& P_{G}=N_{t o t} k T \\
& P_{t u r b}=0.5 N_{t o t} m_{p} \mu \eta^{2} \\
& N_{e}=N_{t o t} /(1 .+1.1 x)
\end{aligned}
$$

I used the equations above in order to build plane-parallel models for the lower transition region of the HR $7428 \mathrm{~K} 2$ primary component. As an estimation of the Volumetric Emission Measure $(V E M)$ vs $T_{\text {eff }}$ of the HR $7428 \mathrm{~K} 2$ primary component I used the measured by Griffiths \& Jordan 1998 for the RS CVn system, HR 1099 primary component $\left(R_{H R 109 K K 1 I V}=3.9 R_{\odot}\right)$, opportunely scaling them, for the bigger radius $\left(R_{K 2}=40 R_{\odot}\right)$ of HR $7428 \mathrm{~K} 2$ star, according the formula $E M_{0.3}=V E M /\left(4 \pi R^{2}\right)$ (Brown et al. 1991) and the parameters of Table 1.

From this estimated $E M_{0.3}$ a grid of 160 transition region models has been built by means of equations 3.1 and 3.2 using a grid of 23 values of electron density at the fixed temperature $T_{e}=50000 \mathrm{~K}$ obtained scaling of a factor from 0.5 up to 30 the values of electron density measured in $\operatorname{HR} 1099\left(N_{e}=5 \cdot 10^{+11}\right)$. For each of these 23 electron density values, a grid of seven values of the turbulent velocity in the layer with $T_{0}=10^{4}$ $v_{\text {turb }}\left(T_{0}=10^{4}\right)=10,20,30,40,50,60,70 \mathrm{kms}^{-1}$ has been considered for the calculation of turbulent velocity distribution according the empirical law by Griffiths \& Jordan98 (1998) $v_{\text {turb }}(T)=v_{\text {turb }}\left(T_{0}\right) *\left(T / T_{0}\right)^{1 / 4}$ between $\log (T)=4.0$ and $\log (T)=5.3$.

These transition region models provide the upper boundaries for the radiative transfer calculations of the chromospheric models, while the adopted photospheric model provides the lower boundaries of the chromospheric models.

\section{The Chromospheric Model}

For each one of the 160 transition region models and for each of nine values of $T_{\text {min }}$ in the range between $\sim 2800 \mathrm{~K}$ and $4200 \mathrm{~K}$ that we have chosen with a step of less than $200 \mathrm{~K}$ as points where to cut the photospheric Kurucz model, a grid of 25 chromospheric models are generated by a smooth spline interpolation between the photosphere and the transition region using as free parameters a grid of $5 \times 5$ interpolation knots. We impose 

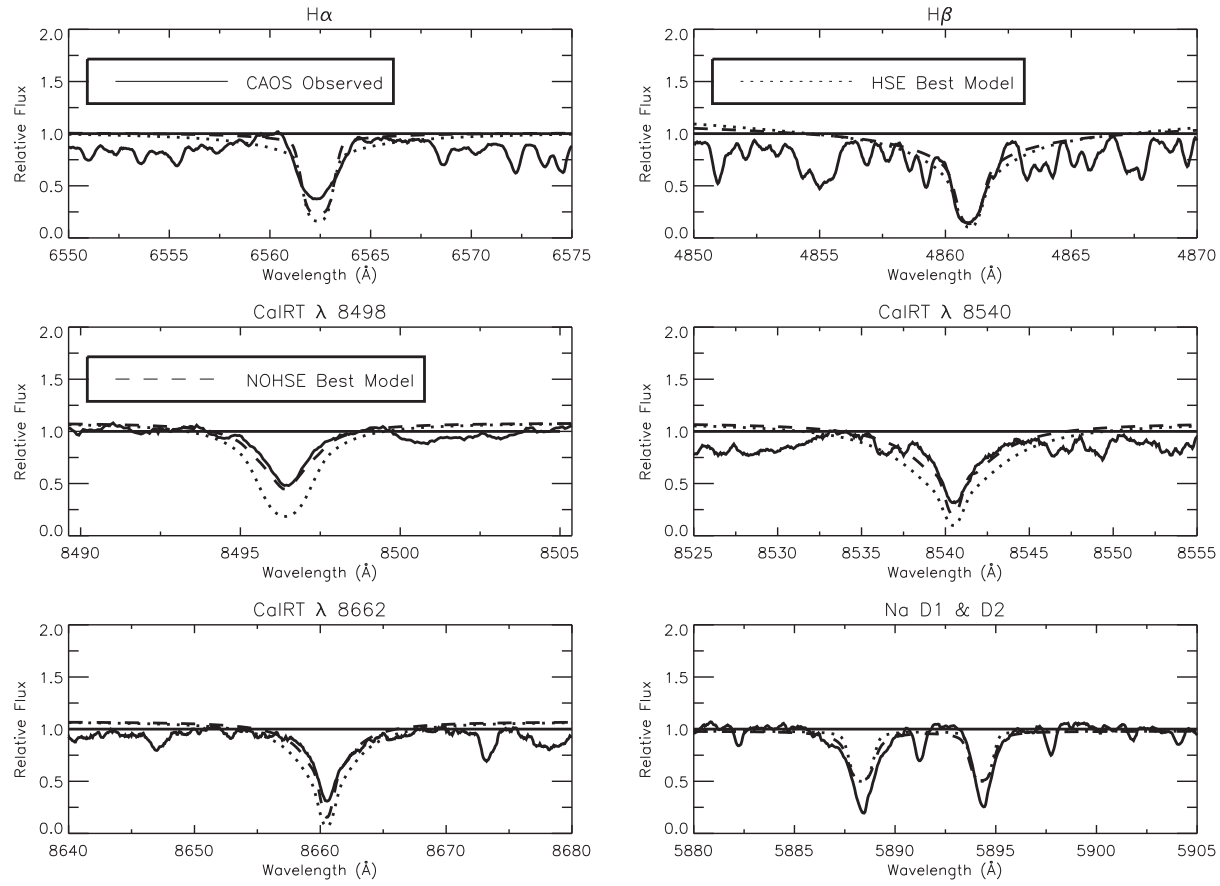

Figure 2. NLTE H- $\alpha$, H- $\beta$, Ca II IRT, Na I D normalized profiles computed for the best $H S E$ (dotted line) and the best NOHSE (dashed line) models compared with observations.

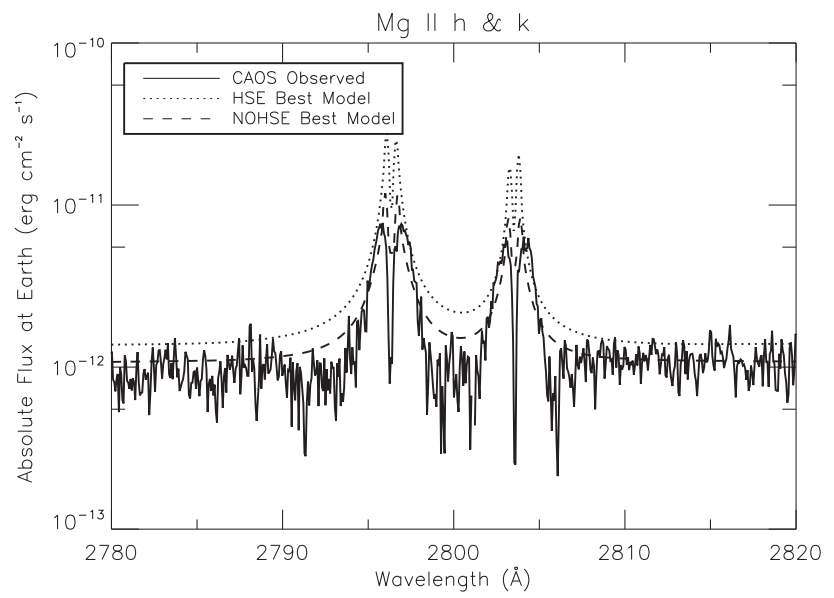

Figure 3. $\mathrm{Mg}$ II h\&k absolute flux profiles computed from the best $H S E$ and the best NOHSE atmospheric models compared with the IUE observation.

the chromospheric structures to have a monotonic temperature dependence on column $\operatorname{mass}(d T / d m \leqslant 0)$.

The final grid of models includes $160 * 9 * 25=36225$ models, and only 15691 satisfy the $d T / d m \leqslant 0)$ constraint and are shown in Fig. 1. 

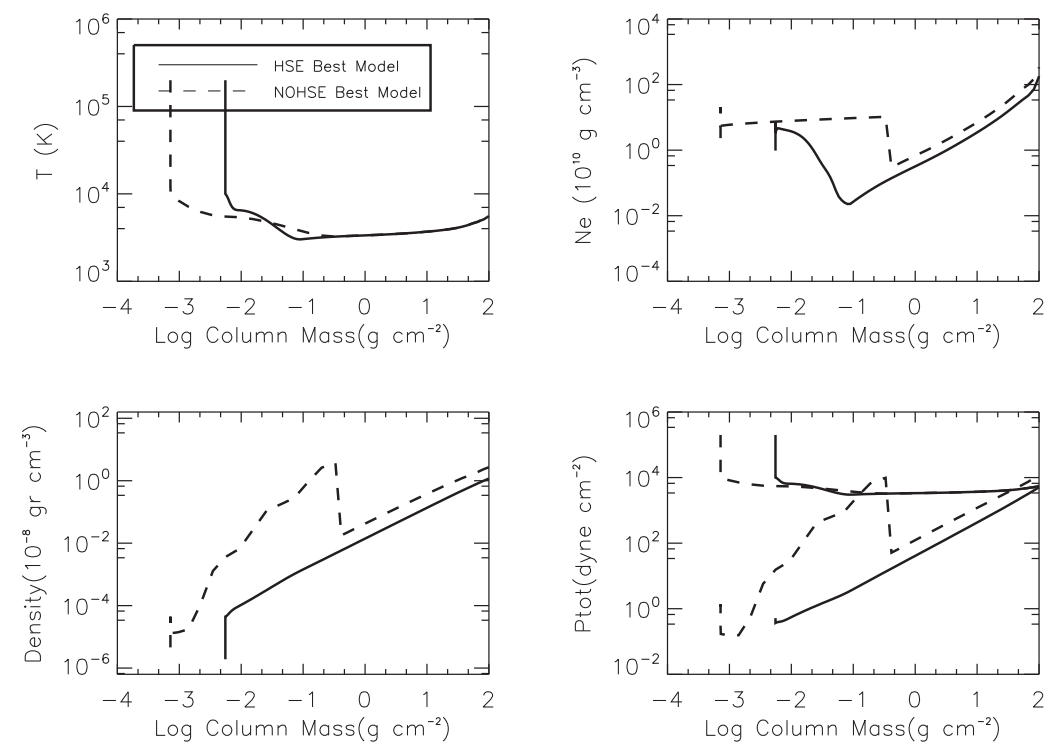

Figure 4. Temperature, electron density, density mass and pressure versus Column Mass for the best $H S E$ and NOHSE models of the K2 primary component of the RS CVn system HR 7428 . In the last plot, the two best models are shown for references.

\section{Computation}

The coupled equations of radiative transfer and statistical equilibrium were solved using the version 2.2 of the code Multi (Carlsson 1986), for the $\mathrm{H}, \mathrm{Ca}, \mathrm{Na}, \mathrm{Mg}$ atomic models. The $\mathrm{H}$ atomic model incorporates 16 states of $\mathrm{H}$, with $84 b$ - $b$ and $9 b$ - $f$ transitions. The Ca atomic model incorporates 8 states of CaI, the lowest 5 states of CaII and the ground state of CaIII, $9 b-b$ and $13 b$ - $f$ transitions are treated in detail. The Mg II atomic model is made of 3 states MgI, the lowest 6 states of MgII and the ground state of mgIII, $9 b$ - $b$ and $9 b$ - $f$ transitions are treated in detail. The Na atomic model incorporates 12 levels: 11 levels of $\mathrm{NaI}$ and the ground state of $\mathrm{NaII}$ and $29 b-b$ and 11 $b-f$ transitions are treated in detail. The opacity package included in the code takes into account free-free opacity, Rayleigh scattering, and bound-free transitions from hydrogen and metals, I included the line blanketing contribution to the opacity using the method described in Busá et al. (2001).

Imposing Hydrostatic Equilibrium - HSE Models

As a first step I imposed hydrostatic equilibrium to the hydrogen. In detail, starting with the LTE hydrogen populations for the electron pressure and density calculation, hydrogen is iterated to convergence. Then Hydrostatic equilibrium $d P_{T o t}=\rho g d h$ is solved and electron pressure and hydrogen populations are updated, the loop continues until we obtain a convergence. Theobtained electron density is then used to solve the $\mathrm{Ca}, \mathrm{Mg}$, and $\mathrm{Na}$ radiative transfer and statistical equilibrium equations. The population densities obtained from the $\mathrm{H}$ calculation are used to obtain the background NLTE source function in the $\mathrm{Ca}, \mathrm{Mg}$, and $\mathrm{Na}$ calculations. This computation modifies the initial grid of Fig. 1 because a new column of electron density is obtained, and we obtain a new grid of only 2052 models that we call HSE models.

\section{Not imposing Hydrostatic Equilibrium - NOHSE Models}

I also considered not imposing hydrostatic equilibrium that means fixing the electron densities to the ones of the original grid of Fig. 1. In this case we consider all the 15691 models that only satisfy the $d T / d m \leqslant 0$ constraint and we call these, NOHSE models. 

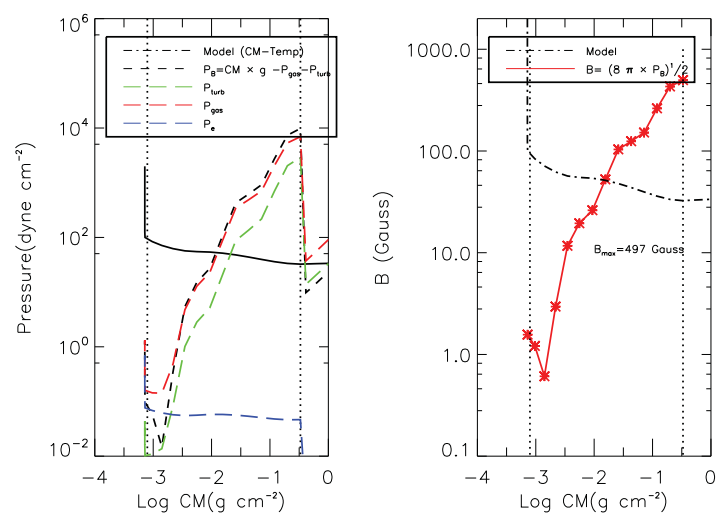

Figure 5. Chromospheric magnetic field distribution obtained considering the whole lacking pressure as magnetic pressure.

This approach takes into account the possibility that some pressure contributions are neglected and becomes a method to derive an estimate of the lacking pressure component. In such an approach I use the electron density as a free parameter, looking for the electron density distribution that best fit the data. I accept as best solution, also a distribution whose total pressure is not balancing the gravity, accepting the hypothesis that a new pressure component could be considered for achieving the equilibrium.

Implications of HSE - NOHSE Models

Both the HSE grid of 2052 models and the initial grid of 15691 model have been considered in the radiative transfer calculations for $\mathrm{H}, \mathrm{Na}, \mathrm{Ca}$ and $\mathrm{Mg}$. For each grid, the models that converge to solution for all the atoms have been taken into account for the comparison with the observed spectrum. The computed profiles of $\mathrm{H}-\alpha, \mathrm{H}-\beta$, Ca II IRT, Na I D, have been convolved for a rotational profile with $v \sin i=17 \mathrm{kms}^{-1}$ (Marino et al. 2001) and an instrumental profile with $\frac{\Delta(\lambda)}{\lambda}=45,000$, normalized and then compared with observations. The computed profiles of $\mathrm{Mg}$ II h\&k have been obtained by the weighted sum of the K2 star profile and the A2 star profile and weighting for the $d^{2} / R^{2}$ factor. Wavelength shifts, to account for orbital velocities, are applied to synthetic spectra.

\section{Results}

I used a $\chi^{2}$ minimization procedure for the selection of the model that best describes the mean outer atmosphere of HR 7428. This procedure has been applied both to the HSE and NOHSE set of models. We find that the best NOHSE model has a $\chi_{T o t}^{2}=1.22$ while the best $H S E$ model has a $\chi_{T o t}^{2}=2.60$. This result let me conclude that the NOHSE best model distribution of temperature, gas pressure, electron and population densities is the best description of the mean outer atmosphere of the K2 star of the binary system HR7428. In Fig. 3, the HSE and the NOHSE best models are compared in temperature, electron density and mass density versus Column Mass (first three plots). But the most important difference between the two models is shown in the last plot of Fig. 3 where the total pressure is plotted versus Column Mass. While, in the HSE model, the total chromospheric pressure is imposed to be equal to the Column Mass multiplied for the gravity, that is PTot $=$ Column Mass $\times \mathrm{g}$, and we obtain a straight line, in the NOHSE model, the total chromospheric pressure exceed the gravity pressure (dashed line is not a straight line but, in the chromospheric layers lays well above of the gravity pressure).

This exceeding pressure has to be balanced by an equal and opposite pressure otherwise we cannot have a stable star. Therefore, I assumed the difference $P_{T o t}-\mathrm{CM} \times \mathrm{g}$ as equal, 
with opposite sign, to the lacking pressure in our calculations, that is: $P_{\text {Totnew }}=P_{\text {turb }}+P_{\text {gas }}+P_{\text {new }}=\mathrm{CM} \times \mathrm{g}$ and therefore $P_{\text {new }}=\mathrm{CM} \times \mathrm{g}-P_{\text {Tot }}$.

I make the hypothesis that the additive pressure, could be a magnetic pressure that from the base of the chromosphere decrease toward the outer layers. This is quite probable in the case of cool active stars. The atmospheres of cool active stars are, in fact, permeated by magnetic fields that emerge from deeper layers. With increasing height we might expect the structure to be more greatly influenced by magnetic fields, since the energy density of the magnetic fields should fall off more slowly than the energy density of the gas (this is the case of solar atmosphere where $\beta=8 \pi N K T_{e} /|(B)|^{2}$ is $\geqslant 1$ in photosphere and $\ll 1$ in transition region layers). In Fig. 4 the new pressure component is shown in comparison to electron pressure, gas pressure, turbulent pressure. It is clear that this pressure component is not negligible being of the same order of strength of the gas pressure. In the hypothesis that the additive pressure, could be a magnetic pressure, I calculated $\mathrm{a} \approx 500$ gauss magnetic field corresponding magnetic field that, from the base of the chromosphere decrease toward the outer layers. It is worthwhile to notice that the NOHSE refers only to the chromospheric layers, therefore this new component pressure estimation refers only to this layer.

\section{References}

Andretta, V., Busá I., Gomez, M. T., \& Terranegra, L. 2005 Astronomy $\&$ Astrophysics 430, 669

Byrne, P. B., Abdul Aziz, H., Amado, P. J., et al. 1998 A\&AS 127, 505

Brown, A. et al. 1991 Astrophysical Journal 373, 614B

Busá I., Andretta, V., Gomez, M. T., \& Terranegra, L. 2001 Astronomy E Astrophysics 373, 993

Carlsson, M. 1986, Technical report 33, Uppsala Astronomical Observatory

Cram, L. E. \& Mullan, D. J. 1979, Astrophysical Journal 234, 579

Fontenla, J. M., Avrett, E. H., \& Loeser, R. 1993, Astrophysical Journal 406, 319

Gratton, L. 1950, Astrophysical Journal 111, 31

Griffiths, N. W. \& Jordan, C. 1998, Astrophysical Journal497, 883

Jordan, C. \& Brown, A. 1981 SPSS, 199J

Hall, D. S., Gessner, S. E. Lines, H. C., \& Lines, R. D. 1990 Astronomical Journal 100, 2017

Harper, G. M. 1992 Monthly Notices of the Royal Astronomical Society 256, 37

Houdebine, E. R. 19960 IAUS 176, 547H

Kalkofen, W., Ulmschneider, P., \& Avrett, E. H. 1999 Astrophysical Journal 521, 141

Kurucz, R. L. 1993, IAU Coll. 138, ASP Conf. Ser. 44, 87

Kurucz, R. L. \& Avrett, E. H. 1981, SAOSR, 391

Leone, F., Avila, G., Bellassai, G., et al. 2016, AJ, 151, 116L

Marino, G., Catalano, S., Frasca, A., \& Marilli, E. 2001, Astronomy \& Astrophysics 375, 100

Mauas, P. J. D. \& Falchi, A. 1994, Astronomy \& Astrophysics 281, 129

Mauas, P. J. D., Falchi, A., Pasquini, L., \& Pallavicini, R. 1997, Astronomy \&3 Astrophysics 326, 249

Mauas, P. J. D., Cacciari, C., \& Pasquini, L. 2006, Astronomy \& Astrophysics 454, 609

Cram, L. E. \& Mullan, D. J. 1979, Astrophysical Journal 234, 579

Parsons, S. B. \& Ake, T. B. 1987, Bull. Am. Astron. Soc. 19, 708

Short, C. I. \& Doyle, J. G. 1998, Astronomy \& Astrophysics 336, 613

Spanó P., Leone, F., Bruno, P., Catalano, S., Martinetti, E., Scuderi, S. 2006, MSAIS 9, 481S

Spanó P., Leone, F., Scuderi, S., Catalano, S., \& Zerbi, F. M. 2004, SPIE, 5492, $373 \mathrm{~S}$

Uitenbroek, H. 1992, ASPC 26, 546

Vernazza, J. E., Avrett, E. H., \& Loeser, R. 1973, Astrophysical Journal 184,605V

Vernazza, J. E., Avrett, E. H., \& Loeser, R. 1981, Astrophysical Journal Supplement 45,635 